\title{
AVIFAUNA NA UNIVERSIDADE DO VALE DO TAQUARI EM LAJEADO - RS
}

\author{
Cleberton Diego Bianchini ${ }^{1}$, Felipe Kuhn², Hamilto Cézar Zanardi Grillo
}

Resumo: As aves são consideradas como bioindicadoras das condições ambientais pois são sensíveis às mudanças e respondem rapidamente a distúrbios em seu habitat. $\mathrm{O}$ Brasil é um país privilegiado pela diversidade de ambientes impactando positivamente na biodiversidade, possuindo, até o momento, 1.919 espécies de aves conhecidas sendo que no RS tem-se o registro de 661 espécies. No entanto, estudos referentes às ocorrências regionais da avifauna no Vale do Taquari ainda são escassos. O objetivo, do presente estudo, foi inventariar a avifauna no campus da Univates e contribuir para o conhecimento regional da avifauna ocorrente no Estado. A metodologia utilizada para o levantamento foi a de transecto e pontos de escuta durante os períodos vespertinos e matutinos, além de amostragens noturnas. O período de amostragem ocorreu de setembro de 2014 a novembro de 2015 de forma direta ou indireta nos variados ambientes do campus, com avaliação posterior do status de conservação das espécies. Os resultados indicaram a ocorrência de 145 espécies, distribuídas em 42 famílias, sendo a Thraupidae e Tyranidae, as mais representativas. A única espécie enquadrada em algum grau de extinção foi a espécie Amazona pretrei (papagaio charão), na categoria Vulnerável - VU. Com a realização do estudo, foi possível perceber que a área, apesar de possuir ambientes antropizados, apresenta boa riqueza de espécies. O estudo também contribuiu para o conhecimento da avifauna da região e Estado.

Palavras-chave: Aves. Riqueza. Extinção.

\section{INTRODUÇÃO}

As aves são sensíveis as mudanças e respondem rapidamente a distúrbios em seu habitat, sendo consideradas bioindicadores das condições ambientais (LINDENMAYER; MARGULES; BOTKIN, 2000). A presença ou

1 Engenheiro Ambiental, Organização Não Governamental ECOBÉ, bianchini@ecobe.org.br.

2 Biólogo, Mestrando em Biotecnologia, Licenciatura pela Univates, felipe_kuhn@hotmail.com.

3 Biólogo, Doutorando do Programa de Pós-Graduação em Ambiente e Desenvolvimento da Univates, Mestre em Biologia Animal, UFRGS. Professor do Curso de Ciências Biológicas da Univates. 
ausência de algumas espécies de aves, bem como as características, interações ecológicas e o status de conservação daquelas presentes permitem até mesmo inferir sobre fatores abióticos de uma área (MOHR, 2012).

O Brasil é um país privilegiado pela diversidade de ambientes impactando positivamente na biodiversidade. Sua avifauna apresenta, até o momento, 1.919 espécies de aves conhecidas (PIACENTINI, 2015). No Rio Grande do Sul tem-se o registro de 661 espécies de aves (BENCKE et al., 2010). Entretanto, estudos referentes as ocorrências regionais da avifauna no Vale do Taquari ainda são escassos. Neste sentido, o objetivo deste estudo é inventariar a avifauna no campus da Univates e contribuir para o conhecimento regional da avifauna ocorrente no Estado.

\section{METODOLOGIA}

\section{1 ÁREA DE ESTUDO}

A Universidade do Vale do Taquari, tratada no presente estudo simplesmente como Univates, está inserida no perímetro urbano do município de Lajeado, Rio Grande do Sul. Segundo o Banco de Dados Regional - BDR (2015) a Univates possui uma área total de $566.262,59 \mathrm{~m}^{2}$, na qual estão localizados 24 prédios que somam 78.645,09 $\mathrm{m}^{2}$ de área construída.

O campus da Univates está localizado na porção centro/nordeste do município de Lajeado, conforme pode ser observado na figura 1 . Sendo que o município está inserido na porção baixa do Vale do Taquari, situado na margem direita do Rio Taquari. Lajeado apresenta $90 \mathrm{~km}^{2}$ de área e 78.446 habitantes, segundo estimativa do IBGE para 2015, apresentando densidade demográfica de 793,07 hab $/ \mathrm{km}^{2}$. O município de Lajeado está inserido nos domínios do Bioma Mata Atlântica e fitoecologicamente está representado pela Floresta Estacional Decidual e Floresta Ombrófila Mista. O local onde está inserida a Univates, constitui-se de área urbana consolidada ou em expansão.

A área de estudo compreende toda a área do campus, sendo um polígono de 56,63 hectares. O campus apresenta áreas abertas com vegetação rasteira, interior de mata, borda de fragmentos, margem de rio, áreas alagadas e estacionamentos com circulação de pessoas. 
Figura 1: Localização da Univates no município de Lajeado

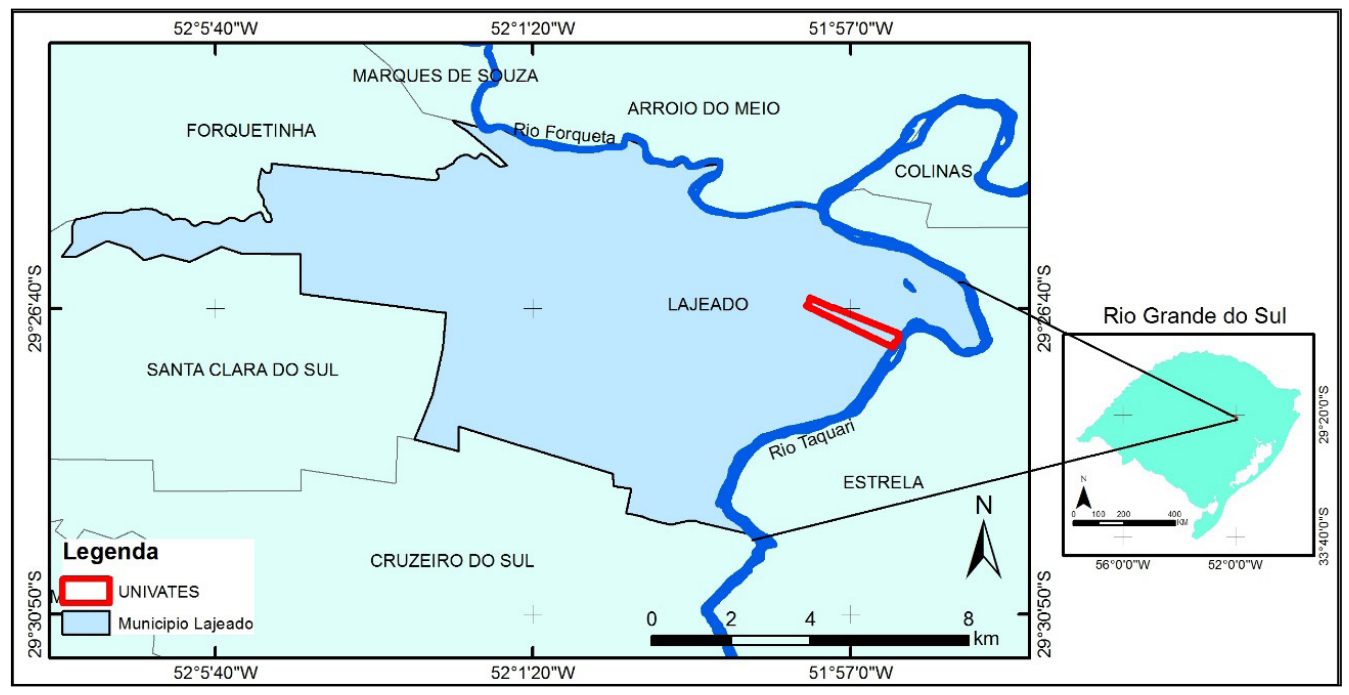

Fonte: IBGE e DSG digitalizadas por HASENACK e WEBER (2010) elaborado por Cleberton Diego Bianchini.

\subsection{MATERIAL E MÉTODOS}

Durante o período compreendido entre setembro de 2014 até novembro de 2015, realizou-se um levantamento qualitativo das espécies encontradas no Campus. O levantamento ocorreu em períodos vespertinos e matutinos, além de amostragens noturnas, quando se utilizou a técnica de play-back para facilitar a identificação de aves com hábitos noturnos. O presente levantamento foi realizado através de observações diretas, com auxílio de binóculos e câmeras digitais, e observações indiretas através das vocalizações, sendo que quando não identificadas no local eram feitos registros de áudio utilizando um gravador Sony PX312 combinado com bioacústica e, posteriormente, um microfone Yoga HT81.

As amostragens ocorreram em caminhadas seguindo transectos em trilhas já existentes além de pontos de escuta em locais privilegiados com duração variada. As amostragens ocorreram em variados ambientes do campus, locais com áreas abertas, interior de mata, bordas de fragmentos, margens do Rio Taquari e em áreas mais antropizadas de estacionamentos e entre os prédios. Para a identificação direta em campo utilizou-se o guia Narosky e Yzurieta (2010), para identificação posterior com fotografia utilizouse o mesmo guia, além de Perlo (2009), Souza (2002), Sigrist (2013) e também auxílio de sites especializados, como Wikiaves, página do The Cornell Lab of Ornithology (Neotropical Birds) e Eco Registros. 
A classificação taxonômica e nomenclatura das espécies registradas seguem conforme Lista de Aves do Brasil do Comitê Brasileiro de Registros Ornitológicos (PIACENTINI, 2015). O status de conservação foi verificado conforme o Decreto Estadual n 51.797 de 8 de setembro de 2014 que declara as espécies da fauna silvestre ameaçadas de extinção no Estado do Rio Grande do Sul.

\section{RESULTADOS E DISCUSSÕES}

Foram registradas 145 espécies de 42 famílias nos ambientes vistoriados, conforme pode ser observado na Tabela 1. Esse número de espécies representa $22 \%$ das 661 espécies de aves registradas para estado do Rio Grande do Sul conforme Bencke (2010). As famílias mais representativas foram a Thraupidae e Tyranidae, com 17 e 16 espécies, respectivamente.

Tabela 1: Lista de espécies de aves registradas no Campus do Centro Universitário UNIVATES no município de Lajeado, no período de setembro de 2010 a dezembro de 2015

\begin{tabular}{ll}
\hline NOME DO TÁXON & \multicolumn{1}{c}{ NOME COMUM } \\
\hline Cracidae & aracuã-escamoso \\
\hline Ortalis squamata (Lesson, 1829) & \\
\hline Ardeidae & socó-boi \\
\hline Tigrisoma lineatum (Boddaert, 1783) & savacu \\
\hline Nycticorax nycticorax (Linnaeus, 1758) & socozinho \\
\hline Butorides striata (Linnaeus, 1758) & garça-vaqueira \\
\hline Bubulcus ibis (Linnaeus, 1758) & garça-branca \\
\hline Ardea alba Linnaeus, 1758 & maria-faceira \\
\hline Syrigma sibilatrix (Temminck, 1824) & \\
\hline Threskiornithidae & caraúna \\
\hline Plegadis chihi (Vieillot, 1817) & tapicuru \\
\hline Phimosus infuscatus (Lichtenstein, 1823) & \\
\hline Cathartidae & urubu-de-cabeça-vermelha \\
\hline Cathartes aura (Linnaeus, 1758) & urubu de cabeça preta \\
\hline Coragyps atratus (Bechstein, 1793) & \\
\hline Accipitridae & gavião-tesoura \\
\hline Elanoides forficatus (Linnaeus, 1758) & sovi \\
\hline Ictinia plumbea (Gmelin, 1788) & gavião-carijó \\
\hline Rupornis magnirostris (Gmelin, 1788) & gavião-de-cauda-curta \\
\hline Buteo brachyurus Vieillot, 1816 & saracura-do-mato \\
\hline Rallidae & \\
\hline Aramides saracura (Spix, 1825) & \\
\hline
\end{tabular}


Charadriidae

Vanellus chilensis (Molina, 1782)

quero-quero

Jacanidae

Jacana jacana (Linnaeus, 1766)

jaçanã

\section{Columbidae}

Columbina talpacoti (Temminck, 1810)

rolinha

Columbina picui (Temminck, 1813)

rolinha-picuí

Columba livia Gmelin, 1789

pombo-doméstico

Patagioenas picazuro (Temminck, 1813)

pombão

Zenaida auriculata (Des Murs, 1847)

pomba de bando

Leptotila verreauxi Bonaparte, 1855

juriti-pupu

Leptotila rufaxilla (Richard \& Bernard, 1792)

juriti gemedeira

Cuculidae

Piaya cayana (Linnaeus, 1766)

alma-de-gato

Crotophaga ani Linnaeus, 1758

anu-preto

Guira guira (Gmelin, 1788)

anu-branco

Tapera naevia (Linnaeus, 1766)

saci

Tytonidae

Tyto furcata (Temminck, 1827)

coruja de igreja

Strigidae

Megascops choliba (Vieillot, 1817)

corujinha-do-mato

Athene cunicularia (Molina, 1782)

coruja-buraqueira

Nyctibiidae

Nyctibius griseus (Gmelin, 1789)

mãe da lua

\section{Apodidae}

Chaetura meridionalis

andorinhão-do-temporal

\section{Trochilidae}

Florisuga fusca (Vieillot, 1817)

beija-flor-preto

Anthracothorax nigricollis (Vieillot, 1817)

beija-flor-de-veste-preta

Stephanoxis lalandi (Vieillot, 1818)

beija-flor-de-topete-verde

Chlorostilbon lucidus (Shaw, 1812) besourinho-de-bico-vermelho

Thalurania glaucopis (Gmelin, 1788) beija-flor-de-fronte-violeta

Hylocharis chrysura (Shaw, 1812)

\section{Trogonidae}

Trogon surrucura (Vieillot, 1817)

beija-flor-dourado

\section{Alcedinidae}

Megaceryle torquata (Linnaeus, 1766)

surucuá-variado

Chloroceryle amazona (Latham, 1790)

martim-pescador-grande

Chloroceryle americana (Gmelin, 1788) martim-pescador-verde martim-pescador-pequeno 
Ramphastidae

Ramphastos dicolorus (Linnaeus, 1766)

tucano-de-bico-verde

\section{Picidae}

Picumnus temminckii (Lafresnaye, 1845)

Melanerpes candidus (Otto, 1796)

Veniliornis spilogaster (Wagler, 1827)

Colaptes melanochloros (Gmelin, 1788)

Colaptes campestris (Vieillot, 1818)

Celeus flavescens (Gmelin, 1788)

\section{Falconidae}

Caracara plancus (Miller, 1777)

Milvago chimachima (Vieillot, 1816)

Micrastur semitorquatus (Vieillot, 1817)

Falco femoralis (Temminck, 1822)

\section{Psittacidae}

Myiopsitta monachus (Boddaert, 1783)

picapauzinho-de-coleira

pica-pau-branco

picapauzinho-verde-carijó

pica-pau-verde-barrado

pica-pau-do-campo

pica-pau-de-cabeça-amarela

Amazona pretrei (Temminck, 1830)

Amazona aestiva (Linnaeus, 1758)

Thamnophilidae

Dysithamnus mentalis (Temminck, 1823)

Thamnophilus caerulescens (Vieillot, 1816)

carcará

carrapateiro

falcão-relógio

falcão-de-coleira

Mackenziaena leachii (Such, 1825)

Conopophagidae

Conopophaga lineata (Wied, 1831)

caturrita

papagaio-charão

papagaio verdadeiro

\section{Dendrocolaptidae}

Xiphorhynchus fuscus (Vieillot, 1818)

choquinha-lisa

choca-da-mata

Lepidocolaptes falcinellus (Cabanis \& Heine, 1859)

Dendrocolaptes platyrostris (Spix, 1825)

borralhara-assobiadora

\section{Furnariidae}

Furnarius rufus (Gmelin, 1788)

joão-de-barro

Lochmias nematura (Lichtenstein, 1823) joão-porca

Syndactyla rufosuperciliata (Lafresnaye, 1832)

trepador-quiete

Schoeniophylax phryganophilus (Vieillot, 1817)

bichoita

Synallaxis ruficapilla (Vieillot, 1819)

pichororé

Synallaxis cinerascens Temminck, 1823

pi-puí

Synallaxis spixi (Sclater, 1856)

joão-teneném

Cranioleuca obsoleta (Reichenbach, 1853)

arredio-oliváceo

\section{Pipridae}

Chiroxiphia caudata (Shaw \& Nodder, 1793)

tangará 
Platyrinchidae

Platyrinchus mystaceus (Vieillot, 1818) patinho

Rhynchocyclidae

Mionectes rufiventris (Cabanis, 1846)

abre-asa-de-cabeça-cinza

Leptopogon amaurocephalus (Tschudi, 1846)

cabeçudo

Phylloscartes ventralis (Temminck, 1824)

borboletinha-do-mato

Tolmomyias sulphurescens (Spix, 1825)

bico-chato-de-orelha-preta

Poecilotriccus plumbeiceps (Lafresnaye, 1846)

tororó

\section{Tyrannidae}

Hirundinea ferruginea (Gmelin, 1788)

Camptostoma obsoletum (Temminck, 1824)

Elaenia flavogaster (Thunberg, 1822)

Elaenia parvirostris (Pelzeln, 1868)

Serpophaga subcristata (Vieillot, 1817)

Legatus leucophaius (Vieillot, 1818)

Pitangus sulphuratus (Linnaeus, 1766)

gibão-de-couro

Machetornis rixosa (Vieillot, 1819)

risadinha

guaracava-de-barriga-amarela

Myiodynastes maculatus (Statius Muller, 1776)

guaracava de bico curto

Megarynchus pitangua (Linnaeus, 1766)

alegrinho

bem-te-vi-pirata

bem-te-vi

suiriri-cavaleiro

Tyrannus melancholicus (Vieillot, 1819)

Tyrannus savana (Daudin, 1802)

bem-te-vi-rajado

neinei

suiriri

Empidonomus varius (Vieillot, 1818)

tesourinha

Myiophobus fasciatus (Statius Muller, 1776)

peitica

Lathrotriccus euleri (Cabanis, 1868)

filipe

Satrapa icterophrys (Vieillot, 1818)

enferrujado

Vireonidae

Cyclarhis gujanensis (Gmelin, 1789)

Vireo chivi (Vieillot, 1817)

suiriri-pequeno

\section{Hirundinidae}

Pygochelidon cyanoleuca (Vieillot, 1817)

pitiguari

juruviara

Stelgidopteryx ruficollis (Vieillot, 1817)

andorinha-pequena-de-casa

Progne tapera (Vieillot, 1817) andorinha-serradora

Progne chalybea (Gmelin, 1789)

andorinha-do-campo

\section{Troglodytidae}

Troglodytes musculus (Naumann, 1823)

andorinha-grande

\section{Turdidae}

Turdus leucomelas (Vieillot, 1818)

Turdus rufiventris (Vieillot, 1818)

Turdus amaurochalinus (Cabanis, 1850)

corruíra

sabiá-branco

sabiá-laranjeira

sabiá-poca 
NOME DO TÁXON

Turdus subalaris (Seebohm, 1887)

Turdus albicollis (Vieillot, 1818)

Mimidae

Mimus saturninus (Lichtenstein, 1823)

Motacillidae

Anthus lutescens (Pucheran, 1855)

\section{Passerellidae}

Zonotrichia capensis (Statius Muller, 1776)

Ammodramus humeralis (Bosc, 1792)

\section{Parulidae}

Setophaga pitiayumi (Vieillot, 1817)

Geothlypis aequinoctialis (Gmelin, 1789)

Basileuterus culicivorus (Deppe, 1830)

Myiothlypis leucoblephara (Vieillot, 1817)

\section{Icteridae}

Cacicus chrysopterus (Vigors, 1825)

Cacicus haemorrhous (Linnaeus, 1766)

Icterus pyrrhopterus (Vieillot, 1819)

Agelaioides badius (Vieillot, 1819)

Molothrus bonariensis (Gmelin, 1789)

\section{Thraupidae}

Pipraeidea melanonota (Vieillot, 1819)

Pipraeidea bonariensis (Gmelin, 1789)

Tangara sayaca (Linnaeus, 1766)

Tangara preciosa (Cabanis, 1850)

Sicalis flaveola (Linnaeus, 1766)

Hemithraupis guira (Linnaeus, 1766)

Volatinia jacarina (Linnaeus, 1766)

Trichothraupis melanops (Vieillot, 1818)

Coryphospingus cucullatus (Statius Muller, 1776)

Tersina viridis (Illiger, 1811)

Dacnis cayana (Linnaeus, 1766)

Coereba flaveola (Linnaeus, 1758)

Sporophila caerulescens (Vieillot, 1823)

Saltator similis (d'Orbigny \& Lafresnaye, 1837)

Poospiza nigrorufa (d'Orbigny \& Lafresnaye, 1837)

Microspingus cabanisi Bonaparte, 1850

Pyrrhocoma ruficeps (Strickland, 1844)

\section{NOME COMUM}

sabiá-ferreiro

sabiá-coleira

sabiá-do-campo

caminheiro-zumbidor

tico-tico

tico-tico-do-campo

mariquita

pia-cobra

pula-pula

pula-pula-assobiador

tecelão

guaxe

encontro

asa-de-telha

chupim

saíra-viúva

sanhaço-papa-laranja

sanhaço-cinzento

saíra-preciosa

canário-da-terra

saíra-de-papo-preto

tiziu

tiê-de-topete

tico-tico-rei

saí-andorinha

saí-azul

cambacica

coleirinho

trinca-ferro

quem-te-vestiu

quete-do-sul

cabecinha-castanha 


\begin{tabular}{ll}
\hline NOME DO TÁXON & NOME COMUM \\
\hline Cardinalidae & \\
\hline Habia rubica (Vieillot, 1817) & tiê-de-bando \\
\hline Cyanoloxia brissonii (Lichtenstein, 1823) & azulão \\
\hline Fringillidae & \\
\hline Spinus magellanicus (Vieillot, 1805) & pintassilgo \\
\hline Euphonia chlorotica (Linnaeus, 1766) & fim-fim \\
\hline Euphonia pectoralis (Latham, 1801) & gaturamo-bandeira \\
\hline Chlorophonia cyanea (Thunberg, 1822) & ferro-velho \\
\hline Passeridae & \\
\hline Passer domesticus (Linnaeus, 1758) & pardal \\
\hline
\end{tabular}

Fonte: dos autores (2017)

Destaca-se o registro de três indivíduos de Amazona pretrei sobrevoando o campus no sentido oeste para leste/nordeste, ao entardecer. Salienta-se que a espécie Amazona pretrei (papagaio charão) está enquadrado na categoria Vulnerável - VU a nível estadual (RIO GRANDE DO SUL, 2014), nacional (BRASIL, 2014) e mundial (IUCN, 2008). Com exceção da $A$. pretrei, não se observou outras espécies com algum grau de ameaça a nível Estadual e Nacional.

Bica et al. (2014) realizaram um levantamento da avifauna na Univates em que encontraram 114 espécies. Salienta-se que estes autores encontraram 10 espécies que não foram encontradas no presente levantamento, sendo: Aramides cajanea, Pyrhura frontalis, Picumnus nebulosos, Elaenia mesoleuca, Myiarchus swainsoni, Tangara seledon, Stephanophorus diadematus, Poospiza lateralis, Sicalis luteola e Pseudoleistes virescens. As ausências das cinco primeiras espécies mencionadas, podem estar relacionadas ao maior tempo de levantamento do estudo comparado ao presente. Quanto as outras cinco espécies, cabe destacar que a espécie Tangara Seledon não ocorre na região de Lajeado (BirdLife International and Nature Serve, 2014; Agpaoa, Janelle, and Burns, 2012), possivelmente os autores tenham confundido no momento da identificação em campo com a Tangara preciosa que é muito comum na região de Lajeado e não consta no trabalho; Poospiza lateralis (Sibley and Monroe 1990, 1993; Stotz et al. 1996) foi dividido em $P$. lateralis e $P$. cabanisi em 2009, possivelmente os autores confundiram Poospiza lateralis, que possui ocorrência no sudeste do País, com Poospiza cabanisi (Bonaparte, 1850), que possui ocorrência para a região em questão; Pseudoleites virecens é uma espécie que habita campos e pântanos (SICK, 1997; NAROSKY, 2010) e não ocorre nesta região segundo BirdLife International and Nature Serve, 2014, possivelmente os autores tenham confundido com o Pseudoleistes guirahuro (chopim do brejo) que não consta no trabalho; Stephanophorus diadematus habita áreas elevadas sendo umas das aves mais típicas dessas áreas (SICK, 1997; SIGRIST, 2013); Sicalis luteola habitam e 
frequentam áreas de campos limpos e capinzais, podendo ser secos ou úmidos ( SICK, 1997; SIGRIST, 2013);

Oliveira e Kohler (2010) realizaram um levantamento da avifauna da RPPN da Unisc, localizada no município de Sinimbu - RS, por um período de dois anos de amostragem. O levantamento apresentou uma lista contendo 169 espécies de aves para o local. O levantamento na RPPN da UNISC apresentou tempo de amostragem maior além de ter sido realizado em área rural. No entanto, os resultados encontrados no presente estudo são positivos, visto que a área em questão encontra-se em área urbana e o tempo de amostragem foi menor do que o referido estudo.

MOHR (2010) avaliou a distribuição, diversidade e composição da riqueza de aves em ambientes de Floresta Ombrófila Mista em uma área localizada em Soledade/RS. Também avaliou as respectivas áreas de borda e matriz, analisando a distribuição da abundância e guildas alimentares de aves nestes ambientes e as variáveis da paisagem que influenciavam a distribuição da abundância e riqueza. A autora analisou nove fragmentos em suas áreas de matriz sendo que as amostras ocorreram em pontos, durante 10 minutos e sem raio definido. As amostragens ocorreram em duas ocasiões na primavera de 2011 e em duas ocasiões no verão de 2012. Neste trabalho foram identificadas 125 espécies sendo que a maior riqueza e abundância ocorreram nas áreas de matriz em comparação com outros ambientes avaliados.

Bianchini (2015) realizou um levantamento no Morro Gaúcho, no município de Arroio do Meio. O levantamento ocorreu em três dias diferentes e totalizaram 12 horas de amostragem. O autor utilizou o método de transecto, percorrendo as trilhas já existentes, intercalando com pontos de escuta sem raio e tempo definido. O trabalho identificou 128 espécies, revelando o grande potencial do local para avifauna.

\section{CONCLUSÕES}

A área estudada sofre com a pressão do crescimento urbano da cidade de Lajeado. O campus da Univates abriga diferentes paisagens, sendo que a área mais preservada se encontra na margem do Rio Taquari. No presente estudo foram encontradas 145 espécies de aves, atestando a importância destas áreas, mesmo que fragmentadas e antropizadas, para a manutenção da diversidade biológica. O presente estudo contribui para o conhecimento da avifauna regional, uma vez que estudos desta natureza ainda são escassos na região. O estudo também contribui para o conhecimento sobre a distribuição das espécies a nível Estadual e Nacional. 


\section{REFERÊNCIAS}

AGPAOA, Jenelle, and BURNS, Kevin J. 2012. Green-headed Tanager (Tangara seledon), Neotropical Birds Online (T. S. Schulenberg, Editor). Ithaca: Cornell Lab of Ornithology; retrieved from Neotropical Birds Online: http:/ / neotropical.birds. cornell.edu/portal/species / overview?p_p_spp $=611276$

BENCKE, Glayson A.; DIAS, Rafael A.; BUGONI, Leandro; AGNE, Carlos E.; FONTANA, Carla S.; MAURICÍO, Giovana N.; MACHADO, Diogenes B.; Revisão e atualização da lista das aves do Rio Grande do Sul, Brasil. Iheringia, v. 100, n.4, p. 519-556, 2010.

BIANCHINI, Cleberton Diego. "Determinação de áreas mais indicadas para implantação de unidades de conservação no Vale do Taquari - RS". 2015. Monografia (Graduação em Engenharia Ambiental) - Centro Universitário UNIVATES, Lajeado, nov. 2015. Disponível em: <http://hdl.handle.net/10737/993>. Acesso em: 20 maio 2016.

BICA, Jonas Bernardes et al. AVIFAUNA NO CAMPUS DO CENTRO UNIVERSITÁRIO UNIVATES, LAJEADO, RIO GRANDE DO SUL, BRASIL. Caderno de Pesquisa, v. 26, n. 1, p. 29-35, 2014.

BIRDLIFE INTERNATIONAL AND NATURE SERVE (2014) Bird Species Distribution Maps of the World. 2012. Tangara seledon. The IUCN Red List of Threatened Species. Version 2015-4

BIRDLIFE INTERNATIONAL AND NATURE SERVE (2014) - Bird Species Distribution Maps of the World. 2012. Pseudoleistes virescens. The IUCN Red List of Threatened Species. Version 2015-4

ECO REGISTROS - Registros Ecológicos de la Comunidad. Disponível em http:/ / www.ecoregistros.org/site/index.php. Acessado em diversos momentos no decorrer do desenvolvimento do estudo.

HASENACK, Heinrich; WEBER, Eliseu. Base cartográfica vetorial contínua do Rio Grande do Sul-escala 1: 50.000. Porto Alegre: UFRGS-IB-Centro de Ecologia, v. 1, 2010.

INTERNATIONAL UNION FOR CONSERVATION OF NATURE - IUCN. The IUCN Red List of Threatened Species. Versão 2015. Disponível em:< http:/ / www.iucnredlist. org/ > . Acesso em 20 de dezembro de 2015.

LINDENMAYER, David, B.; MARGULES, Chris R.; BOTKIN, Daniel B. Indicators of biodiversity for ecologically sustainable forest management. Conservation Biology, v. 14, n. 4, p. 941-950, 2000.

MINISTÉRIO DO MEIO AMBIENTE-MMA. Portaria MMA n. 444/2014, de 17 de dezembro de2014. Diário Oficial da União, Brasília, DF, v. 126, n. 245, p. 121, 18 dez. 2014. Seção 1. 
MOHR, Luciane R. da S. Distribuição da avifauna em ambientes fragmentados de Floresta Ombrófila Mista na Bacia Hidrográfica do Rio Forqueta, RS, Brasil. 2013.

NAROSKY, Tito. Aves de Argentina y Uruguay: Birds of Argentina \& Uruguay. guía de identificación edición total: a field guide total edition. / Tito Narosky y Dario Yzurieta. - 16 ${ }^{\mathrm{a}}$ ed. - Buenos Aires, Argentina: Vázquez Mazzini Editores, 2010.

PERLO, Ber van. A field guide to the birds of Brazil. Oxford: Oxford University Press, 2009.

PIACENTINI, Vítor de Q. et al. Annotated checklist of the birds of Brazil by the Brazilian Ornithological Records Committee/Lista comentada das aves do Brasil pelo Comitê Brasileiro de Registros Ornitológicos. Revista Brasileira de OrnitologiaBrazilian Journal of Ornithology, v. 23, n. 2, p. 90-298, 2015.

RIO GRANDE DO SUL. Decreto ${ }^{\circ}$ 51.797, de 8 de setembro de 2014. Declara as espécies de fauna silvestre ameaçadas de extinção no Estado do Rio Grande do Sul.

SICK, Helmut. Omitologia brasileira. Rio de Janeiro: Nova Fronteira, 1997.

SIGRIST, Tomas. Guia de campo Avis Brasilis - Avifauna Brasileira / Tomas Sigrist; ilustrado por Tomas Sigrist - São Paulo: Avis Brasilis, 2013.

SOUZA, Deodato. All the birds of Brazil: an identification guide. Salvador: Dall, 2002.

THE CORNELL LAB OF ORNITHOLOGY - Neotropical Birds. Disponível em http:/ / neotropical.birds.cornell.edu/portal/home. Acessado em diversos momentos no decorrer do desenvolvimento do estudo.

WIKIAVES. Disponível em http:/ / www.wikiaves.com.br/. Acessado em diversos momentos no decorrer do desenvolvimento do estudo. 